\title{
AMENDMENTS
}

\section{Author Correction: Metagenomic analysis of colorectal cancer datasets identifies cross-cohort microbial diagnostic signatures and a link with choline degradation}

Andrew Maltez Thomas (D), Paolo Manghi, Francesco Asnicar (D), Edoardo Pasolli, Federica Armanini, Moreno Zolfo (D), Francesco Beghini (D), Serena Manara, Nicolai Karcher, Chiara Pozzi, Sara Gandini (D), Davide Serrano, Sonia Tarallo (D), Antonio Francavilla (D), Gaetano Gallo (D), Mario Trompetto, Giulio Ferrero (D), Sayaka Mizutani, Hirotsugu Shiroma, Satoshi Shiba, Tatsuhiro Shibata (D), Shinichi Yachida, Takuji Yamada, Jakob Wirbel (D), Petra Schrotz-King (D), Cornelia M. Ulrich, Hermann Brenner, Manimozhiyan Arumugam (D), Peer Bork (D), Georg Zeller (D), Francesca Cordero, Emmanuel Dias-Neto (D), João Carlos Setubal, Adrian Tett, Barbara Pardini (iD, Maria Rescigno, Levi Waldron (D), Alessio Naccarati iD and Nicola Segata (D)

Correction to: Nature Medicine https://doi.org/10.1038/s41591-019-0405-7, published online 1 April 2019.

In the version of this article initially published, the affiliation for Chiara Pozzi, Sara Gandini and Davide Serrano was given as the European Institute of Oncology. The correct affiliation is IEO, European Institute of Oncology IRCCS. In addition, the data for age, BMI and sex were missing from Table 1 for the HanniganGD_2018 dataset, and consequently for the totals of all datasets. These data are given in Table 1 here. The errors have been corrected in the HTML and PDF versions of the article.

Table 1 | Size and characteristics of the large-scale CRC metagenomic datasets included in this study

\begin{tabular}{lllllll} 
Dataset & Groups $(\boldsymbol{n})$ & Age $($ mean \pm s.d.) & BMI (mean \pm s.d.) & Sex F(\%)/M(\%) & Country & No. of reads $\left(\times 10^{9}\right)$ \\
\hline $\begin{array}{l}\text { HanniganGD_2018 } \\
\left(\text { ref. }{ }^{31}\right)\end{array}$ & Control (28) & $55.46 \pm 9.4$ & $26.63 \pm 5.1$ & $60.7 / 39.3$ & USA (54), & 0.5 \\
& Adenoma (27) & $61.74 \pm 10.8$ & $27.36 \pm 4.45$ & $40.7 / 59.3$ & Canada (28) \\
Total & CRC (27) & $58.6 \pm 10.9$ & $30.41 \pm 7.5$ & $25.9 / 74.1$ & & \\
& Control (308) & $\mathbf{6 2 . 0 1} \pm \mathbf{3 . 7}$ & $\mathbf{2 5 . 4} \pm \mathbf{1 . 4}$ & $\mathbf{4 3 . 5 / 5 6 . 5}$ & $\mathbf{4 5 . 6}$ \\
& Adenoma (143) & $\mathbf{6 3 . 8 8} \pm \mathbf{1 . 9}$ & $\mathbf{2 6 . 8} \pm \mathbf{1 . 1}$ & $\mathbf{4 0 . 5 / 5 9 . 5}$ & \\
& CRC (313) & $\mathbf{6 4 . 6 7} \pm \mathbf{3 . 8}$ & $\mathbf{2 5 . 8} \pm \mathbf{1 . 7}$ & $\mathbf{3 3 . 5 / 6 6 . 5}$ &
\end{tabular}

Published online: 29 October 2019

https://doi.org/10.1038/s41591-019-0663-4

(c) The Author(s), under exclusive licence to Springer Nature America, Inc. 2019

\section{Author Correction: Reporting guidelines for clinical trials evaluating artificial intelligence interventions are needed}

The CONSORT-AI and SPIRIT-AI Steering Group

Correction to: Nature Medicine https://www.nature.com/articles/s41591-019-0603-3, published online 24 September 2019.

In the version of this article originally published, the name of author Lavinia Ferrante di Ruffano was incorrectly spelled as Lavinia Ferrante Di Ruffano. The error has been corrected in the HTML and PDF versions of the article.

Published online: 12 November 2019

https://doi.org/10.1038/s41591-019-0662-5

๑) Springer Nature America, Inc. 2019 